\title{
Correlation between Child Behavior and Depression in Mother with Autism Spectrum Disorders Child
}

\author{
Kumiko Kido'), Kumi Fujita ${ }^{2)}$ \\ 1)Department of Nursing, Faculty of Health Sciences, Kagawa Prefectural \\ University of Health Science, Kagawa, Japan \\ 2)Faculty of Social Welfare, Yamaguchi Prefectural University, Yamaguchi, Japan
}

\section{ABSTRACT}

Background: The behavioural characteristics of an autistic child can lead to challenging relationships with the mother. For mothers who serve as the key person in the care of their children with ASD, maintaining good mental health, which may be directly related to the growth and development of their children, is an important issue for professionals engaged in parenting support. The aim of this study was to determine the interaction between the behavioural problems of children with ASD (from toddlers to adolescents) and their mothers' depression.

Subjects and Method: This was a crosssectional study carried out in Yamaguchi Prefecture, Japan. The recruitment of subjects was through a paediatric out-patient unit. Eighty-six mothers with ASD participated in this observational study. The Center for Epidemiological Studies-Depression (CES-D) scale-was used as a measure of depression in mothers, and the Child Behaviour Checklist (CBCL) was used as an assessment of the children's behavioral characteristics. The prevalence of depression among mothers of children with ASD was estimated and related factors were also examined.

Results: There were 66 boys (76.7\%), 12 girls (14.0\%) and 8 (9.3\%) non-responders. Comparing the pre- adolescent and adolescent groups, there were significant differences in the ages of mothers and children $(\mathrm{P}<0.001)$. In this study, the prevalence rate of depression in mothers of children with ASD was $47.7 \%$ (95\% $\mathrm{CI}=36.8$ to 58.7 ), and the prevalence rate of depression in mothers of adolescent children group was $48.6 \%$ ( $95 \% \mathrm{CI}=31.4$ to 6.0 ). The predictive factors of maternal depression were others' problems (toileting problems, and masturbation) in CBCL and children's age group.

Conclusion: These children's behavioural characteristics were recognised as serious problems by their mothers, which in turn can negatively affect their mental health, particularly the depression of mothers in the adolescent group was probably due to concerns about their children's unsocial behaviour.

Keywords: Mental health, behavioural problems, pre-adolescent, adolescent

\section{Correspondence:}

Kumiko Kido, Department of Nursing, Faculty of Health Sciences, Kagawa Prefectural University of Health Sciences, Murechohara 281-1 Takamatsu, Kagawa, Japan. Email: kkumikoo305@gmail.com. Mobile:+819013360452

Cite this as:

Kido K, Fujita K (2021). Correlation between Child Behavior and Depression in Mother with Autism Spectrum Disorders Child. J Matern Child Health. 06(04): 475-485. https://doi.org/10.26911/thejmch.2021.06.04.09.

(c) (1) J (2) Journal of Maternal and Child Health is licensed under a Creative Commons Attribution-NonCommercial-ShareAlike 4.0 International License.

\section{BACKGROUND}

Autism spectrum disorder (ASD) is often characterised by a deficiency in socialemotional reciprocity, imperfect verbal and nonverbal communication, and repetitive motor movements and is a neurodevelopmental disorder (Diagnostic and Statistical Manual of Mental Disorders [DSM] 5) 
(American Psychiatric Association, 2013). The behavioural characteristics of an autistic child can lead to challenging relationships with the mother, as she often is the closest carer (Gobrial, 2018). Some evidence suggests that such mothers are more anxious than the fathers(Pisula and Porębowicz-Dörsmann, 2017), and it has been reported that their children's problems that stem from their unique behavioral characteristics are negatively associated with the mental well-being of mothers (Zablotsky et al., 2013 ; Kousha et al., 2016; Jahan et al., 2020). A recent study has revealed that the mothers of children with developmental disabilities in Japan, in which approximately half of them, suffer from depressive symptoms although it is not the mothers of children with ASDs (Kido \& Fujita, 2019).

The possibility of the decrease in parents' self-efficacy can be attributed to the lack of appropriate services for their children when the latter reach adolescence (Weiss et al., 2016). According to Kido \& Fujita (2019), mothers of children with developmental disabilities (although not exclusively an ASD) in adolescence were apprehensive that their children will develop sexual interests and desire that could lead to sexual victimisation or abuse (Kido \& Fujita, 2019). It is possible that sexual development of children with autism is related to unarticulated apprehensiveness rather than acceptability of mothers. In Japan, few studies have focused on the association between the mental well-being of mothers of children with ASD and children's behavioural characteristics in which the latter is attaining secondary sexual characteristics.

Generally, poor maternal mental health has negative effects on children, such as neglect (Ayers et al., 2019; Wolford et al., 2019). Similarly, for mothers who serve as the key person in the care of their children with ASD, maintaining good mental health, which may be directly related to the growth and development of their children, is an important issue for professionals engaged in parenting support.

This study aimed to determine whether the psychological well-being of mothers of children with ASDs in Japan deteriorates when their children reach adolescents with ASD and further clarify whether behavioral characteristics of children with ASDs, especially each domain of the CBCL, are related to their mothers' mental health.

\section{SUBJECTS AND METHOD}

\section{Study Design}

This was a cross-sectional study. The mothers of children with ASD who visited a paediatrician for their children's consultations in a rural city (Yamaguchi Prefectre in Japan) were asked to enrol in this study. The survey was conducted between early September to the end of November 2011.

\section{Population and Sample}

The participants were recruited through a paediatrician that the mother visited with the child at paediatric outpatients'unit in a hospital.Most participants were mothers of children diagnosed with Asperger's syndrome or autism based on the DSM version IV at the time of the study. The DSM was revised to version 5 in 2013 (published Japanese version) and changed the name of the diagnosis to ASD. In this study, the diagnosis of children was ASD. In total, 86 mothers of children with ASD have agreed to participate in this study.

\section{Study Variables}

In this study, the multiple liner regression Analysis was performed to examine the predictive factors of mental health status of mothers of children with ASD. The maternal CES-D was used as dependent variable and each items of CBCL, child's sex 
and child's age group as independent variables. The CES-D consists of a 20-items self-assessment questionnaire for depressive symptoms. As a dependent variable, the raw score of each mother's CES-D was used. The CBCL consists of a 113 items regarding children's emotional and behavioral problems. For each CBCL items, I to VIII and 'others' problems' were included as independent variables. The raw scores of each CBCL were used as independent variables. The children's sex was a categorical variable with Boy or Girl, which was coded for quantification. The children's age was the continuous variable, however the age groups of the children was divided into adolescent and pre-adolescent based on the age of 10 .

\section{Operational Definition of Variables}

Child's sex was quantified because of the categorical valiable. Boy coded as 1; Girl coded as 0 .

Child's age was divided into two groups. According to the WHO definition, Adolescent group: children aged $>10$ years coded as 1; Pre-adlescent group: children aged $<10$ years coded as 0 .

The maternal CES-D utilized raw scores, which were continuous variables, without coding.

\section{Study Instruments}

The basic attributes of the subjects were age of the mother and child and the sex of the child. The Centre for Epidemiologic Studies Depression Scale (CES-D) was used as a measure of the mother's mental health, whereas the CBCL was used as an assessment of the children's behavioural characteristics.

CES- D was used as the measure of the mental health status of mothers of children with ASD. It was developed by the National Institute of Mental Health (USA) as a mental health screening tool. CES-D, which is internationally standardised, is a 20-item self-assessment questionnaire for depressive symptoms, ranging from 'rarely or none of the time (less than 1 day)' to 'most or all of the time (5-7 days)'. The responses were rated using a 4-point Likert scale (o-4), with scores ranging from 0 to 6o. Typically, a score $\geq 16$ is the cut-off point, which was identified as 'depressed' (Tuovinen et al., 2018).

The Child Behaviour Checklist (CBCL) is a scale developed to assess children's emotional and behavioural problems. The measure is constructed using the average Tscores of the internalising broadband, externalising broadband and total problem scale. It has been used in the assessment of children with developmental disabilities and in various studies (Havdahl et al., 2016; Rescorla et al., 2019).

In Japan, the Japanese versions of CBCL/2-3, Youth Self-report for Ages/1118and CBCL/4-18 for were published based on the 1991 version and have been used in a large number of studies (Kamo et al., 2021). In this study, we also used the Japanese version of CBCL according to the target age groups. All items in the CBCL can be divided into eight areas and 'other problems'. The eight areas of assessment were as follows: I, withdrawal-depression; II, somatic complaints; III, anxiety/ depression; IV, social problems; V, thinking problems; VI, attention problems; VII, delinquent behaviour; and VIII, aggressive behaviour. 'Others' problems' include 'parorexia', 'masturbation' etc. All items are rated on a scale of $0-2$. The total score is calculated as the T-score. I to III are then converted into $\mathrm{T}$-scores on the introversion scale (as internalising problems), whereas VII and VIII are converted into T-scores on the extraversion scale (as externalising problems). $\mathrm{T}$ scores of 70 are considered clinically significant for all scales (Aitken et al., 2019). A total score $\leq 66$ is determined 
Kido et al./ Child Behavior and Depression in Mother with Autism Spectrum Disorders Child

as the normal zone, $<70$ as the borderline zone and $\geq 70$ as the clinical zone. To determine the balance between the internalising and externalising problem scales, the T-score on the internalising problem scale was divided by the T-score on the externalising problem scale. The balance between $\mathrm{T}$-scores on the introversion and extraversion scales was closer to 1 , which further indicates a balance between the internalising and externalising problems.

\section{Data Analysis}

To examine the association between behavioral characteristics of the child's age and their mothers' mental health status, we divided the children into two groups: preadolescent group and adolescent group. Referring to the definition of the World Health Organisation (WHO), children aged $>10$ years were defined as the adolescent group; children aged $<10$ years were defined as the pre-adolescent group.

Multiple linear regression Analyses were conducted with maternal CES-D as the dependent variable and items of the
CBCL, the child's age group (adolescent/ pre-adolescent) and sex (boy/girl) as independent variables. Mann-Whitney U-test was also performed for comparison between two groups of Pre-adolescent and Adolescent for the mother's age, child's age and each items of the CBCL.

\section{Research Ethics}

We explained to the mothers that they would not be disadvantaged in their children's medical treatment or support if they refused to participate in the study. This study was approved by the ethical committee of Yamaguchi Prefectural University. The ethical approval number was 21-13.

\section{RESULTS}

\section{Attributes of the subjects}

The sex of the children in the pre-adolescent and adolescent groups, normal/ depressive category by the CES-D and ratio of normal/borderline/clinical zone by the CBCL are shown in Table 1.

Table 1. Basic characteristics of the subjects

\begin{tabular}{|c|c|c|c|c|c|c|c|c|c|}
\hline \multirow[b]{2}{*}{ Characteristics } & \multicolumn{3}{|c|}{ Total } & \multicolumn{3}{|c|}{ Pre-adolescent } & \multicolumn{3}{|c|}{ Adolescent } \\
\hline & $\mathbf{n}$ & $\%$ & 95\%CI (\%) & $\mathbf{n}$ & $\%$ & 95\%CI (\%) & $\mathbf{n}$ & $\%$ & 95\%CI (\%) \\
\hline \multicolumn{10}{|l|}{ Child's sex } \\
\hline Boys & 66 & 76.7 & 66.4 to 85.2 & 37 & 72.5 & 58.3 to 84.1 & 29 & 82.9 & 3.2 to 26.7 \\
\hline Girls & 12 & 14.0 & 7.4 to 23.1 & 8 & 15.7 & 7.0 to 28.6 & 4 & 11.4 & 3.2 to 26.7 \\
\hline NA & 8 & $9 \cdot 3$ & 4.1 to 17.5 & 6 & 11.8 & 4.4 to 23.9 & 2 & $5 \cdot 7$ & 0.7 to 19.2 \\
\hline \multicolumn{10}{|l|}{ CES-D } \\
\hline Normal & 45 & 52.3 & 41.3 to 63.2 & 27 & 52.9 & 38.5 to 67.1 & 18 & 51.4 & 34.0 to 68.6 \\
\hline Depression & 41 & $47 \cdot 7$ & 36.8 to 58.7 & 24 & 47.1 & 32.9 to 61.5 & 17 & 48.6 & 31.4 to 66.0 \\
\hline \multicolumn{10}{|l|}{ CBCL } \\
\hline Normal zone & 32 & 37.2 & 27.0 to 48.3 & 17 & $33 \cdot 3$ & 20.8 to 47.9 & 15 & 42.9 & 26.3 to 60.6 \\
\hline Border zone & 12 & 14.0 & 7.4 to 23.1 & 10 & 19.6 & 9.8 to 33.1 & 2 & $5 \cdot 7$ & 0.7 to 19.2 \\
\hline Clinical zone & 42 & 48.8 & 37.9 to 59.9 & 24 & 47.1 & 32.9 to 61.5 & 18 & 51.4 & 34.0 to 68.6 \\
\hline
\end{tabular}

CES-D: Center for Epidemiologic Studies Depression Scale

CBCL: Child Behavior Checklist

CI:confidence interval

NA: no answer 
Kido et al./ Child Behavior and Depression in Mother with Autism Spectrum Disorders Child

The mean age (SD) and 95\% confidence interval (CI) of the mothers and children are shown in Table 2. The Mann-Whitney U-test showed a significant difference in the age of mothers and children in the pre-adolescent and adolescent groups $(\mathrm{P}<0.001)$ (Table 2). The mother's age was significantly higher in the adolescence groups. The age difference between the two groups was approximately 7 years. Regarding the child's sex, there were 66 boys (76.7\%), 12 girls (14.0\%) and 8 (9.3\%) nonresponders. There were five times as many boys as girls.
Moreover, the prevalence rate of depression in mothers of children with ASD was $47.7 \%$ (95\% CI $=36.8$ to 58.7 ), and 41 mothers (44.7\%) were determined to have depression as per the CES-D. The prevalence rate of depression in mothers of adolescent children group was 17 mothers (48.6\%). The clinical zone of CBCL in the pre-adolescent group was 24 children (47.1\%) and in the adolescent group 18 children (51.4\%). The assessment of CBCL completed by the mothers is shown in Table 3 .

Table 2.The comparison of pre-adolescence and adolescent -mothers' age and children's age

\begin{tabular}{lccccccc}
\hline & \multicolumn{2}{c}{ Total } & \multicolumn{2}{c}{ Pre-adolescent } & \multicolumn{2}{c}{ Adolescent } & \multirow{2}{*}{ p } \\
\cline { 2 - 7 } Categories & Mean & SD & Mean & SD & Mean & SD & \\
\hline Mother's age & 40.3 & 5.4 & 37.5 & 4.8 & 44.0 & 3.7 & $<0.001$ \\
Child's age & 9.3 & 4.3 & 6.3 & 2.1 & 13.8 & 2.3 & $<0.001$ \\
\hline
\end{tabular}

\section{CES-D and CBCL}

The mean CES-D score was (Mean= 16.970; $\mathrm{SD}=9.239$ ) (95\% CI= 14.99 to 18.95). There was no significant difference in CES-D scores between the pre-adolescent and adolescent groups $(\mathrm{P}=0.619)$ (See Table 3$)$.

Table 3. The comparison of pre-adolescent and adolescent- CES-D and CBCL

\begin{tabular}{|c|c|c|c|c|c|c|c|}
\hline & \multicolumn{2}{|l|}{ Total } & \multicolumn{2}{|c|}{ Pre-adolescent } & \multicolumn{2}{|c|}{ Adolescent } & \multirow{2}{*}{$\mathbf{p}$} \\
\hline & Mean & SD & Mean & SD & Mean & SD & \\
\hline CES-D & 16.9 & 9.2 & 16.7 & 8.3 & 18.1 & 10.5 & 0.619 \\
\hline CBCL I & 3.6 & 2.7 & $3 \cdot 5$ & 2.6 & 3.8 & 2.9 & 0.743 \\
\hline CBCL II & 1.9 & 2.5 & 1.6 & 2.5 & 2.2 & 2.5 & 0.078 \\
\hline CBCL III & 7.5 & 5.0 & 7.0 & $4 \cdot 3$ & 8.3 & 5.8 & 0.286 \\
\hline CBCL IV & 6.9 & 2.4 & 6.7 & 2.2 & 7.1 & 2.6 & 0.372 \\
\hline CBCL V & 3.2 & 2.8 & 2.8 & 2.4 & 3.8 & 3.1 & 0.144 \\
\hline CBCL VI & 10.3 & $3 \cdot 7$ & 10.1 & $3 \cdot 3$ & 10.7 & 4.2 & 0.293 \\
\hline CBCL VII & 3.4 & 2.8 & 3.2 & 2.3 & $3 \cdot 7$ & 3.4 & 0.929 \\
\hline CBCL VIII & 11.2 & 6.8 & 11.3 & 6.4 & 11.0 & 7.5 & 0.761 \\
\hline $\begin{array}{l}\text { Others' } \\
\text { problems }\end{array}$ & 12.8 & 6.8 & 13.9 & 6.3 & 11.2 & $7 \cdot 3$ & 0.064 \\
\hline $\begin{array}{l}\text { Internal } \\
\text { T score }\end{array}$ & 66.0 & 9.4 & 64.0 & 8.3 & 68.5 & 8.5 & 0.116 \\
\hline $\begin{array}{l}\text { External } \\
\text { T score }\end{array}$ & 64.7 & 8.6 & 63.1 & $7 \cdot 5$ & 66.0 & 6.3 & 0.070 \\
\hline $\begin{array}{l}\text { Total } \\
\text { T score }\end{array}$ & 68.5 & 8.2 & 68.6 & 7.1 & 68.5 & 9.6 & 0.772 \\
\hline $\begin{array}{l}\text { Internal } \\
\text { T/External T } \\
\text { (balance) }\end{array}$ & 1.0 & 0.1 & 1.0 & 0.1 & 1.0 & 0.1 & \\
\hline
\end{tabular}


Kido et al./ Child Behavior and Depression in Mother with Autism Spectrum Disorders Child

The mean CBCL scores showed that internalising problem T-score was 66.05 \pm 9.473 (95\% CI= 64.02 to 68.08), externalising problem scale T-score was 64.70 $($ Mean $=64.70 ; \mathrm{SD}=8.638)(95 \% \mathrm{CI}=62.85$ to 66.55) and balance (internalising problem T-scores/externalising problem Tscores) was 1.03 (Mean= 1.03; $\mathrm{SD}=0.157)$ (95\% $\mathrm{CI}=0.99$ to 1.06). Although no significant difference was observed in the CBCL scores between the pre-adolescent and adolescent groups, the CBCL II (somatic complaint) tended to be more frequent in the adolescent group $(\mathrm{P}=0.078)$, 'others' problems' tended to be more frequent in the pre-adolescent group $(\mathrm{P}=0.064)$, and the External $\mathrm{T}$ score (combination of rulebreaking behaviour and aggressive beha- vior) tended to be higher in the adolescent group $(\mathrm{P}=0.070)$. As 'others' problems' in CBCL, the mothers reported children's toileting problems and playing with one's genital (genital touching).

\section{Predictive factors for CES-D}

The multiple regression analysis was performed with CES-D as dependent variable and each CBCL domains in the scale and child's sex and children's age group as independent variables (See Table 4). The predictive factors of CES-D that showed significant differences were 'others' problems' in one of CBCL domains ( $\beta=$ $0.480, p=0.002$ ) and child's age group (pre-adolescent and adolescent) $(\beta=0.230$, $\mathrm{P}=0.044)$.

Table 4 .The results of regression model-The predictive factors for CES-D of mothers

\begin{tabular}{lcc}
\multicolumn{1}{c}{ Independet Variables } & Estimate( $\boldsymbol{\beta})$ & $\mathbf{p}$ \\
\hline CBCLI & -0.10 & 0.952 \\
CBCLII & -0.27 & 0.051 \\
CBCLIII & 0.36 & 0.070 \\
CBCLIV & -0.16 & 0.304 \\
CBCLV & -0.25 & 0.163 \\
CBCLVI & 0.20 & 0.219 \\
CBCLVII & -0.25 & 0.220 \\
CBCLVIII & 0.25 & 0.178 \\
Others'problems & 0.48 & 0.002 \\
Child's sex (Boys: 1, Girls: o) & -0.08 & 0.488 \\
Child's age group & 0.23 & 0.044 \\
(adolescent : 1,pre-adolescent : o) & & \\
\hline
\end{tabular}

Multiple R-squared: 0.325

$\mathrm{N}$ observation $=86$

\section{DISCUSSION}

The present study examined whether the mental health of mothers of children with ASD in Japan deteriorates when the children with ASD reach adolescence. In addition, we examined whether the behavioral characteristics of children with ASD, specifically the domains of the CBCL, are related to maternal mental health.
In this study, the prevalence rate of depression in mothers of children with ASD was $47.7 \%$ (95\% $\mathrm{CI}=36.8$ to 58.7 ). It was identified that approximately half of the mothers of children with ASD had depression. Costa et al. (2017) reported that parents of children with ASD had increased stress in daily life and their mental wellbeing could be negatively affected. Further, 
the magnitude of stress carers experience could be more likely to increase if their children's disabilities were severe (Rauf et al., 2018). The clinical zone of the CBCL was in the majority at $51.4 \%$ in this study. Although this present study did not measure the severity of ASD, nor the presence or severity of mental retardation of the children, around half of the mothers showed depressed symptoms. Pisula and Porębowicz-Dörsmann (2017) stated that mothers of children with autism are more likely to be stressed than fathers. The mothers of children with ASD are the closest caregivers to these children; thus, they receive the most burden in daily parenting of their children.In relation to the factors associated with maternal d

Depression, the predictive factors of maternal depression were 'others' problems' in CBCL and child's age group (preadolescent and adolescent) in this study. Regarding the details of what mothers identify as difficulties in raising children, as other problematic behaviours, toileting problem and 'masturbation'. These problems could be stressful for the mother and affect their psychological health. Leader et al. (2018) reported that adolescent autistic children have a number of problems regarding defeca-tion, which can often lead to mothers' apprehension. Yet in the present study, scores of other problems tended to be higher in the pre-adolescent group, suggesting that mothers' distresses related to their children's sexual habit and toileting problem are not specific to adolescence. In addition, the children's sexual habit and toileting problem can become a distressing issue for mothers even their children have not reached adolescence. Mothers of pre-adolescent children need support and advice to help them cope with these issues significant to maintain mental well-being (Gobrial, 2018) stated that such obsessions or compulsive behaviors of children with ASD often result in challenging relationships between the mother and child. However, even in a different country, the challenge of parenting children with ASD has unchanged, and support for mothers' children with developmental disabilities has remained a challenge regardless of the country.

Genital touching as 'other problems', which is an unfavourable child's sexual habit for the mothers from an early age, could lead to serious maternal apprehension about the difficulty of dealing with their children's sexual development. A meta-analysis on the sexual development of children with ASD was conducted by Pecora et al. (2016). They reported that the increase in sexual interest in female children can experience experience more adverse sexual experiences than males. Whereas, in our study, approximately 70\% of the children were male.

A qualitative study by Pryde \& Jahoda (2018) has revealed that mothers of male children with intellectual disabilities in adolescence and young adulthood recognise that their son's sexual development is important; however, they remain apprehensive in providing sex education in fear that it would only increase their sons' sexual interest. It is possible that children's sexual development during adolescence negatively affects the mental health of their mothers, becoming one of the biggest stressors. In this study, the prevalence rate of depression in mothers of adolescent children group was $48.6 \%$ (95\% CI $=31.4$ to 66.0 ). This number is higher than in the pre-adolescent group. However, the study did not examine the implementation of sex education, nor was it able to establish that developing sexual interests were a cause of maternal depression. The study showed no evidence that the problems associated with adoles- 
cent children's sexual development had an impact on mothers' mental health negatively. Rather, the CBCL II associated with rulebreaking and aggressive behaviours tends to be higher in the adolescent group, and the depression of mothers in the adolescent group was probably due to concerns about their children's unsocial behavior. Moreover, the mean age of mothers of adolescent group was (Mean $=44.0 ; \mathrm{SD}=3.7$ ) years, which implies that they are on the point of approaching menopause. When mothers of adolescent children enter the menopausal stage, these increased depressive symptoms could be a part of the symptoms during menopause caused by declining ovarian function.

It is necessary to explore the planning of training parents to ensure that their children's development is captured and addressed accurately in order to maintain mothers' mental health. Moreover, as children enter adolescence, providing emotional support to mothers is critical in maintaining a healthy childcare environment to help address parents' apprehensions about their children's several issues. In this study, the children were mostly boys (approximately $70 \%$ ); therefore, this study revealed the characteristics of mothers in terms of caring for their sons. Nevertheless, it is possible that mothers' perceptions could vary depending on their child's sex. Thus, identifying what aspects of a child's unique development negatively affect mothers is a must; furthermore, this step is vital to determine what type of support a mother needs.

Although the study did not address the marital relationships of depressed mothers, the mental well-being of mothers of children with ASD is significant not only to foster their relationship with their child but also to appropriate social support a mother needs (Cuzzocrea et al., 2016;
Marsack \& Samuel, 2017). Further, a good relationship with their partner (child's father) has been determined to reduce the stress of parenting of the child with ASD (Timmons et al., 2016; Pisula and Porębowicz-Dörsmann, 2017).

The self-efficacy of mothers has been determined to decrease as it is difficult for them to receive appro-priate services for their children during adolescence (Weiss et al., 2016). There are issues that affect the mental health of mothers in each generation of children, such as the unsocial behavior in adolescent group and sexual habit and excretion in pre-adolescent group. Regardless of the age of children, mothers need to be supported to maintain their self-efficacy.

It is important to provide services that are suitable for the development of the child to maintain their mother's mental well-being. In Japan, it is necessary to develop a system in which professionals can support mothers of children with ASD and assess the mother's parenting environment as appropriate for the child's development.

This present study has several limitations. First, the target sample was only mothers of children with ASD. It is unclear whether the results were distinctive in mothers of children with ASD, as it was not compared with mothers of children with other developmental disabilities. Second, responses to all scales are self-reported and thus are subject to potential bias. Third, examining mothers' mental status was solely limited to their relation with their children's behavioural characteristics; other factors, such as their relationships with family members and the kind of social support they receive, were not explored. Finally, this study was conducted 9 years ago. It is important to interpret the results with caution, as there are current changes 
Kido et al./ Child Behavior and Depression in Mother with Autism Spectrum Disorders Child

in the society's support system for autistic children and their parents.

In this study, the prevalence of depression in mothers of children with ASD was $47.7 \%$ (95\% $\mathrm{CI}=36.8$ to 58.7 ), and the prevalence rate of depression in mothers of adolescent children group was $48.6 \%$ (95\% $\mathrm{CI}=31.4$ to 66.0 ). The predictive factors of CES-D that showed significant differences were 'others' problems' in CBCL and child's age group. As 'others' problems' in CBCL, the mothers reported children's toileting problems and playing with one's genital (genital touching). The masturbation and toileting problems as compulsive behaviors in children with ASD can lead to mothers' apprehension. The depression of mothers in the adolescent group was probably due to concerns about their children's unsocial behaviour. It is possible that these children's behavioral characteristics were recognised as serious problems by their mothers, which in turn can negatively affect their mental health. Whatever the age of the children, providing emotional and technical support to mothers can be critical in maintaining mothers' mental well-being.

\section{AUTHOR CONTRIBUTION}

Kumiko Kido and Kumi Fujita designed the study and wrote the first draft of the manuscript. Kumiko Kido performed the statistical analysis and wrote the protocol of the study. Kumiko Kido managed the literature searches and review the literatures. All authors read and approved the final manuscript.

\section{CONFLICT OF INTEREST}

There was no conflicts of interest.

FUNDING AND SPONSORSHIP

This work was supported by the JSPS KAKENHI Grant Number JP 21592831 (Representative: Kumiko Kido).

\section{ACKNOWLEDGEMENT}

We would like to thank Dr. Hayashi for his cooperation in asking the subjects to participate in this study.

\section{REFERENCE}

Aitken M (2019). Clinical utility of the CBCL Dysregulation Profile in children with disruptive behavior. J Affect Disord. 253:87-95. https://doi.org/10.1016/j.jad.2019.04.034.

American Psychiatric Association (2013). Diagnostic and statistical manual of mental disorders, fifth edition DSM-5). American Psychiatric Association, Arlington.

Ayers S, Bond R, Webb R, Miller P, Bateson $K$ (2019). Perinatal mental health and risk of child maltreatment: A systematic review and meta-analysis. Child Abuse Negl. 98: 104172. https://doi.org/10.1016/j.chiabu.2019.104172.

Costa AP, Steffgen G, Ferring D (2017). Contributors to well-being and stress in parents of children with autism spectrum disorder. Res Autism Spectr Disord. 37: 61-72. https://doi.org/10$.1016 /$ j.rasd.2017.01.007.

Cuzzocrea F, Cuzzocrea F, Murdaca AM, Costa S, Filippello P, Larcan R (2016). Parental stress, coping strategies and social support in families of children with a disability. Child Care Pract. 22(1): 3-19. https://doi.org/ 10.1080/13575279.2015.1064357.

Gobrial E (2018). The lived experiences of mothers of children with the autism spectrum disorders in Egypt. Soc Sci. 7(8). https://doi.org/10.3390/socsci7080133 .

Havdahl KA, von Tetzchner S, Huerta M, Lord C, Bishop SL (2016). Utility of the child behavior checklist as a screener for autism spectrum dis- 
Kido et al./ Child Behavior and Depression in Mother with Autism Spectrum Disorders Child

order. Autism Res. 9(1): 33-42. https://doi.org/10.1002/aur.1515.

Jahan S, Araf K, Gozal D, Griffiths MD, Mamun MA (2020). Depression and suicidal behaviors among Bangladeshi mothers of children with Autism Spectrum Disorder: A comparative study. Asian J Psychiatr. https://doi.org/10.1016/j.ajp.2020.101994.

Kamo T, Ito F, Monden Y, Bussing R, Niwa M, Kawasaki M, Matano M, et al. (2021). Japanese version of the Eyberg child behavior inventory: Translation and validation. Jpn Psychol Res. 12365. https://doi.org/10.1111/jpr.12365.

Kido K, Fujita K (2019). Psychological wellbeing and utmost concern for parenting in mothers of children with developmental disabilities: A qualitative approach using framework matrix. J Heal Care Soc. 29(1): 135-154. https://doi.org/10.4091/iken.2019.002.

Kousha M, Attar HA, Shoar Z (2016). Anxiety, depression, and quality of life in Iranian mothers of children with autism spectrum disorder. J Child Heal Care. 20(3):405-414. https://doi.org/10.1177/1367493515598644.

Leader G, Francis K, Mannion A, Chen J (2018). Toileting problems in children and adolescents with parent-reported diagnoses of autism spectrum disorder. J Dev Phys Disabil. 30(3): 307-327. https://doi.org/10.1007/s10882-018-9587-z.

Marsack CN, Samuel PS (2017). Mediating effects of social support on quality of life for parents of adults with autism. J Autism Dev Disord. 47(8): 23782389. https://doi.org/10.1007/s10803-017-3157-6.

Pecora LA, Mesibov GB, Stokes MA (2016). Sexuality in high-functioning autism: A systematic review and meta-ana- lysis. J Autism Dev Disord. 46(11): 3519-3556. https://doi.org/10.1007/s10803-016-2892-4.

Pisula E, Porębowicz-Dörsmann A (2017). Family functioning, parenting stress and quality of life in mothers and fathers of Polish children with high functioning autism or Asperger syndrome. PLoS One. 12(10). https://doi.org/10.1371/journal.pone.01865 36.

Pryde R, Jahoda A (2018). A qualitative study of mothers' experiences of supporting the sexual development of their sons with autism and an accompanying intellectual disability. Int $\mathrm{J}$ Dev Disabil. 64(3): 166-174. https://doi.org/10.1080/20473869.2018.144 6704.

Rauf NK, Anis-ul-haq M, Khan S (2018). Association of autism child characteristics with maternal and paternal stress. Rawal Med J. 43(2): 263-266. Available at https://www.researchgate.net/profile/Saira-Khan-2/publication/325581707_Association_of_a utism_child_characteristics_with_m aternal_and_paternal_stress/links/5 e76869b92851cf2719d9fcd/Associatio n-of-autism-child-characteristicswith-maternal-and-paternal-stress.pdf

Rescorla LA, Winder-Patel BM, Paterson SJ, Pandey J, Wolff JJ, Schultz RT, Piven J (2019). Autism spectrum disorder screening with the CBCL/ $1^{1 / 2-}$ 5: Findings for young children at high risk for autism spectrum disorder. Autism. 23(1): 29-38. https://doi.org/10.1177/1362361317718482.

Timmons L, Willis KD, Pruitt MM, Ekas NV (2016). Predictors of daily relationship quality in mothers of Children with Autism Spectrum Disorder. J 
Kido et al./ Child Behavior and Depression in Mother with Autism Spectrum Disorders Child

Autism Dev Disord. 46(8). https://doi.org/10.1007/s10803-016-2799-0.

Tuovinen S, Lahti-Pulkkinen M, Girchenko P, Lipsanen J, Lahti J, Heinonen K, Rebecca M, et al. (2018). Maternal depressive symptoms during and after pregnancy and child developmental milestones. Depress Anxiety. 35(8): 732-741. https://doi.org/10.1002/da.22756.

Weiss JA, Tint A, Paquette-Smith M, Lunsky Y (2016). Perceived self-efficacy in parents of adolescents and adults with autism spectrum disorder. Autism. 20(4): 425-434. https://doi.org/10.1177/1362361315586292.
Wolford SN, Cooper AN, McWey LM (2019). Maternal depression, maltreatment history, and child outcomes: The role of harsh parenting. Am J Orthopsychiatry. 89(2): 181191. https://doi.org/10.1037/ortoo00365 .

Zablotsky B, Bradshaw CP, Stuart EA (2013). The association between mental health, stress, and coping supports in mothers of children with autism spectrum disorders. J Autism Dev Disord. 43(6): 1380-1393. https://doi.org/10.1007/s10803-012-16937. 